\title{
Spatial frequencies and the cerebral hemispheres: Contrast sensitivity, visible persistence, and letter classification
}

\author{
DAVID H. PETERZELL and LEWIS O. HARVEY, JR. \\ University of Colorado, Boulder, Colorado \\ and \\ CURTIS D. HARDYCK \\ University of California, Berkeley, California
}

\begin{abstract}
The hypothesis that the two cerebral hemispheres are specialized for processing different visual spatial frequencies was investigated in three experiments. No differences between the left and right visual fields were found for: (1) contrast-sensitivity functions measured binocularly with vertical gratings ranging from 0.5 to 12 cycles per degree (cpd); (2) visible persistence durations for 1- and 10-cpd gratings measured with a stimulus alternation method; and (3) accuracy $\left(d^{\prime}\right)$ and reaction times to correctly identify digitally filtered letters as targets $(\mathrm{L}$ or $\mathrm{H})$ or nontargets ( $T$ or F). One significant difference, however, was found: In Experiment 3, a higher decision criterion $(\beta)$ was used when filtered letters were identified in the right visual field than when they were identified in the left. The letters were filtered with annular, 1-octave band-pass filters with center spatial frequencies of $1,2,4,8$, and $16 \mathrm{cpd}$. Combining four center frequencies with three letter sizes $\left(0.5^{\circ}, 1^{\circ}\right.$, and $2^{\circ}$ high) made some stimuli equivalent in distal spatial frequency (cycles per object) and some equivalent in proximal spatial frequency (cycles per degree). The effective stimulus in the third experiment seemed to be proximal spatial frequency (cycles per degree) not distal (cycles per object). We conclude that each cerebral hemisphere processes visual spatial frequency information with equal accuracy but that different decision rules are used.
\end{abstract}

Considerable experimental evidence indicates that visual information is processed differently in the left and right visual fields. Both accuracy and speed of performance depend on the task, stimulus materials, and visual field of presentation (Friedman \& Polson, 1981; Hellige, 1983; Hellige \& Sergent, 1986; Sergent \& Hellige, 1986). Many believe that these performance asymmetries reflect differences in the way the left and right halves of the brain process information (Bryden, 1982; Hardyck, 1983; Hellige, Corwin, \& Jonsson, 1984; Springer \& Deutsch, 1985). Visual stimuli may be described in terms of their spatial-frequency content, and one factor contributing to hemispheric asymmetry may be that the two cerebral

These experiments were supported in part by grants to $\mathrm{L}$. $\mathrm{O}$. Harvey, Jr., from the National Science Foundation, the Army Research Institute, the Biomedical Research Support Grant Program, National Institutes of Health, and Colorado Video Incorporated, Boulder, Colorado. Some of these results were reported at the Rocky Mountain Psychological Association meeting in Albuquerque, NM, April 30, 1987, and at the Twentieth Annual Mathematical Psychology Meeting, Berkeley, California, August 8, 1987. We thank David Boles, Lyle Bourne, Vincent Di Lollo, Joseph Hellige, Lester Krueger, Joel Norman, and an anonymous reviewer for their comments on earlier drafts of this manuscript. C. D. Hardyck is affiliated with the Departments of Education and Psychology at the University of California, Berkeley. Reprints may be obtained from either D. H. Peterzell or L. O. Harvey, Jr., Department of Psychology, Campus Box 345, University of Colorado, Boulder, CO 80309. hemispheres differ in their abilities to process different ranges of spatial-frequency information (Sergent, 1982b, 1987). Hemispheric asymmetries could occur because, on the one hand, the sensory capacities of the two halves of the brain differ or, on the other hand, the two halves of the brain differ in their abilities to perform cognitive operations on the sensory information present.

We report here three experiments designed to clarify the role of stimulus and task factors in contributing to visual-field differences in the processing of visual information. In Experiments 1 and 2, contrast sensitivity and visible persistence were measured for the two visual fields to assess possible sensory differences. In Experiment 3, letters of the alphabet containing different bands of spatialfrequency information were used in a target-identification task to assess higher level differences between the hemispheres.

\section{EXPERIMENT 1}

The evidence supporting a difference in the sensory capacity between the two hemispheres is weak at best. The contrast-sensitivity function (CSF), that is, detection sensitivity to sine-wave gratings of different spatial frequencies, is a measure of basic visual resolution capacity. Although there is one report that the left and right visual fields differ in their CSFs (Rao, 1979; Rao, Rourke, \& 
Whitman, 1981), most investigators report no differences (Beaton \& Blakemore, 1981; Blake \& Mills, 1979; Delis, Robertson, \& Efron, 1986; Fiorentini \& Berardi, 1984; Kitterle \& Kaye, 1985; Rijsdijk, Kroon, \& van der Wildt, 1980; Rose, 1983; Szelag, Budohoska, \& Koltuska, 1987; Vassilev, Verskaya, Manahilov, Mitov, \& Leushina, 1985). Kitterle (1986) provides a review of this literature. Kitterle and Kaye (1985) measured monocular contrastsensitivity functions in the nasal and temporal hemifields of each eye, and found that the four resulting contrastsensitivity functions were identical except for small nasal-temporal differences. They concluded that the findings of Rao (1979; Rao et al., 1981) were due to differences in nasal-temporal sensitivity rather than hemispheric asymmetry. Nonetheless, we undertook to measure contrast sensitivity in the left and right visual fields.

\section{Method}

Subjects. A complete set of measurements were obtained on D.H.P., the first author, at the University of California, Berkeley. His results were verified with 3 additional volunteers from the Psychology Department at the University of California, Berkeley. All subjects had normal or corrected-to-normal vision.

Apparatus. Vertical sinusoidal gratings were generated by a PDP11 computer and were displayed on a Tektronix Model 650 CRT monitor with $\mathrm{P} 4$ phosphor at a mean luminance of $27.4 \mathrm{~cd} / \mathrm{m}^{2}$. The screen was viewed binocularly from a distance of $200 \mathrm{~cm}$ and was surrounded by a large white mask with a circular aperture that subtended a visual angle of $6^{\circ}$. The luminance of this mask was approximately the same as the mean luminance of the screen. Black fixation spots were placed $1.5^{\circ}$ to the left and right of the screen. When one of these spots was fixated, the visible portion of the screen was centered $4.5^{\circ}$ from the fovea.

Procedure. The subject sat in a dimly lit booth, with his/her head in a chinrest. During each block of trials, the subject first fixated either the right or the left fixation point for $3 \mathrm{~min}$ while a zerocontrast stimulus was displayed on the screen. After this adaptation period, contrast thresholds were measured for seven test spatial frequencies $-0.5,1,2,4,8,10$, and 12 cycles per degree (cpd)-using a two-alternative forced-choice detection paradigm. The two intervals were $200 \mathrm{msec}$ long, and the onset of each interval was signaled by a tone. By pressing one of two buttons, the subject indicated which interval contained the grating. A tone sounded if the response was incorrect. The contrast of the stimulus was controlled from trial to trial, using the PEST staircase procedure (Taylor \& Creelman, 1967), and was set to converge on the contrast that yielded $75 \%$ correct detection. Within each block there were actually 21 such staircases, 3 for each of the seven tested frequencies, and these were randomly interleaved from trial to trial. The block of trials ended when all 21 staircases had converged. At least 17 blocks of such trials were run for each visual field. The selection of visual field varied randomly from block to block. The testing sessions were spread out over a $1 \frac{112}{2}$ month period.

\section{Results and Discussion}

Contrast sensitivity was computed by taking the reciprocal of each threshold contrast, and the geometric mean sensitivity at each of the seven frequencies was calculated separately for each visual field. The logarithm of these mean contrast sensitivities for D.H.P. are plotted in Figure 1. Each data point is the mean of at least 51 threshold measurements, and the standard error of each

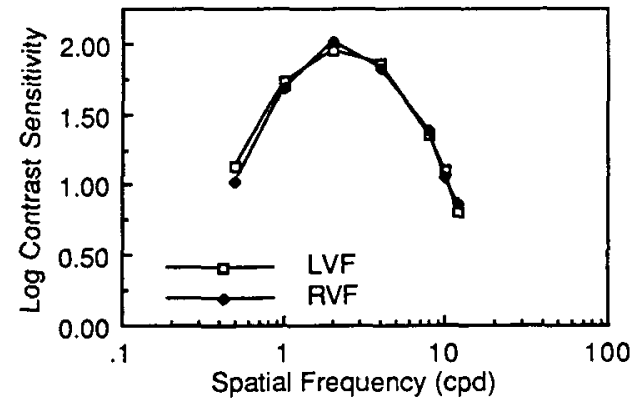

Figure 1. Contrast-sensitivity functions of the left and right visual fields of the first author measured binocularly with vertical sinewave gratings in Experiment 1.

mean is no greater than $0.043 \log$ units. As can be seen in Figure 1, the CSF measured in the left visual field (LVF) was nearly identical to that in the right (RVF). The sensitivity in the LVF was marginally greater $(p=.05)$ than that in the RVF for the lowest spatial frequency of $0.5 \mathrm{cpd}$, and there were no significant differences at the other frequencies. No significant differences between the two visual fields were found in the other 3 observers, and we therefore conclude that there is identical contrast sensitivity in the RVF and LVF. These results confirm the published findings of Fiorentini and Berardi (1984), Kitterle and Kaye (1985), and Rose (1983), and suggest that any nasal-temporal differences in contrast sensitivity balance out when stimuli are viewed binocularly.

We feel confident that the results are not due to eyemovement artifacts: First, there is no reason to suspect that subjects initiated a trial without first establishing proper fixation. Second, the sensitivity to high spatial frequencies found in this experiment is much lower than that found with foveal fixation under identical test conditions. This finding supports our belief that the test gratings were correctly positioned in the peripheral visual field, where high-frequency sensitivity is known to fall off.

\section{EXPERIMENT 2}

Another index of sensory capacity is the length of time that a stimulus is visible after its physical offset. Perhaps the two visual fields differ in the degree of this visible persistence. Di Lollo (1981) presented four $\times$ four dot matrices to subjects' LVFs and RVFs. Fifteen dots appeared sequentially for $1 \mathrm{msec}$ each, with one dot, chosen randomly, missing from the display. Subjects indicated the location of the missing dot. Visible persistence was measured by varying the interval between presentation of successive dots. No persistence differences were found between the two visual fields.

Di Lollo (1981) did not examine the possibility that the visible persistence of high and low spatial frequencies might differ in the LVF and RVF. The visible persistence of different spatial frequencies has been measured for fo- 
veal vision by determining the temporal separation required for the detection of a blank interval between two successive sine-wave gratings (Badcock \& Lovegrove, 1981; Bowling, Lovegrove, \& Mapperson, 1979; Meyer \& Maguire, 1977). We used this method to examine the two visual fields for differences in persistence at different spatial frequencies.

\section{Method}

Subjects. Five male and 5 female undergraduates at the University of California, Berkeley, participated as observers in this experiment to fulfill a course requirement. All had normal or correctedto-normal vision and were right-handed with no reported family history of left-handedness.

Stimuli and Apparatus. Vertical sine-wave gratings of 1.0 and $10.0 \mathrm{cpd}$, and a uniform gray field of equal mean luminance were displayed on the Tektronix monitor used in Experiment 1. They were photographed with Kodak Panatomic X film, and matte prints were prepared so that the stimuli could be presented in a threechannel Scientific Prototype tachistoscope, Model S-1000. These prints were matched for mean reflectance and a contrast of 0.27 . Mean luminance of the stimuli in each channel of the tachistoscope was set at $30.8 \mathrm{~cd} / \mathrm{m}^{2}$ and was calibrated before each subject was tested. The stimuli appeared to the left or right of a red fixation light within rectangular fields, as is shown in Figure 2. The fixation light was continuously visible, and the rest of the display was dark between trials.

Procedure. The subjects adapted in the dark for $3 \mathrm{~min}$ before the experiment began. They then fixated the red dot in the tachistoscope. On each trial, test gratings of identical spatial frequency were simultaneously presented in the LVF and RVF for $75 \mathrm{msec}$. After an interstimulus interval (ISI), the test gratings were again presented in the LVF and RVF for $75 \mathrm{msec}$. During the ISI, one of the following sets of stimuli was flashed: (1) the test grating in the LVF and a gray blank in the RVF; (2) a gray blank in the LVF and the test grating in the RVF; (3) test gratings in both visual fields; or (4) gray blanks in both visual fields. The subject made two judgments about the ISI: whether or not a blank had been presented on the left and whether or not a blank had been presented on the right. Each subject knew that within any block of 12 trials, these presentations were random, with each side having an independent .5 probability of a blank field presentation. A new trial began every $15 \mathrm{sec}$.

Persistence threshold was defined as the ISI that allowed approximately $75 \%$ detection of the blank field. Persistence thresholds were determined separately for each visual field by means of a descending method of limits with a 10-msec step size starting with an ISI

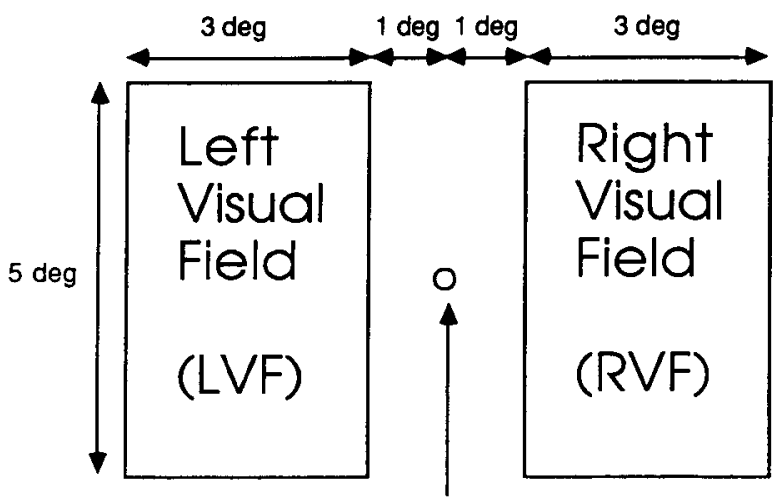

Red fixation point

Figure 2. The stimulus configuration used in Experiment 2.

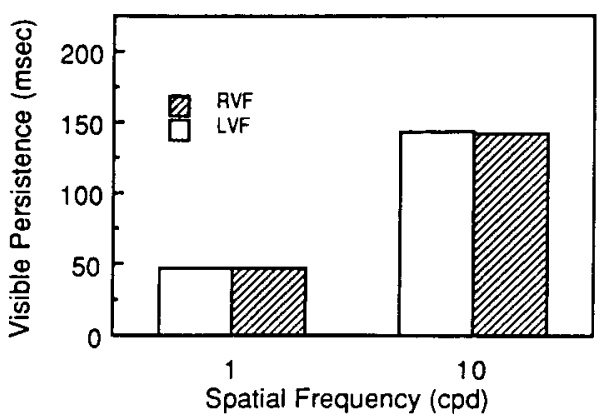

Figure 3. Mean visible persistence, in milliseconds, as a function of spatial frequency for the left (LVF) and right (RVF) visual fields in Experiment 2. Each point is the mean of 10 subjects.

above threshold. Two blocks of 12 trials were presented. If, after the first block, the subject achieved $100 \%$ detection of the blank in both visual fields, the second block was not run. After each set of one or two blocks, ISI was reduced by $10 \mathrm{msec}$. Blocks of trials continued until detection performance fell below $75 \%$ correct in both visual fields. The lowest ISI that produced at least $75 \%$ correct detection of the blank in a specific visual field was recorded as the visible persistence threshold for that visual field.

\section{Results and Discussion}

Visible persistence, in milliseconds, of 1.0- and 10.0-cpd gratings in the LVF and RVF is presented in Figure 3. The influence of spatial frequency and of visual field on persistence was assessed with a $2 \times 2 \times 10$ within-subjects analysis of variance. Persistence was significantly longer for the 10.0 -cpd than for the 1.0-cpd grating $[F(1,9)=11.7, p<.01]$. There was no significant difference between the two visual fields, nor was there a significant visual field $\times$ frequency interaction. These results agree with those of Di Lollo (1981, 1983), who used dots as stimuli. They strengthen the conclusion that differences in the processing of visual stimuli are not based on different degrees of visible persistence in the two visual fields.

Whether these conclusions will hold for shorter and longer exposure durations is an empirical question that has not yet been addressed. Hemispheric asymmetries are almost never reported on simple sensory detection experiments, and Experiment 2 is no exception. Although varying exposure duration has often created visual-field asymmetries in more cognitive tasks, it seems unlikely that changing exposure durations would result in asymmetries on this simple sensory detection task. Finally, although increasing the duration of the stimulus in the central visual field results in a linear decrease in persistence, additional perceptual mechanisms are probably not involved (Bowling \& Lovegrove, 1980). It seems unlikely that varying exposure durations would result in the emergence of visual-field asymmetries.

\section{EXPERIMENT 3}

It is important to distinguish between the spatialfrequency information available from the stimuli in the LVF and RVF and the spatial-frequency information re- 
quired by cognitive processes to successfully complete a task (Hellige, 1983; Hellige \& Sergent, 1986; Sergent, 1983c, 1985; Sergent \& Hellige, 1986). Performance of a task is impaired more in the RVF than in the LVF when a stimulus's luminance or exposure duration is reduced, or when retinal eccentricity or blur is increased, or when a stimulus is low-pass filtered (see Jonsson \& Hellige, 1986, and Sergent \& Hellige, 1986, for reviews). Because these stimulus manipulations all reduce the effective highspatial-frequency information available in the visual stimulus, these results imply that low spatial frequencies are processed more rapidly and accurately in the LVF than in the RVF, and that high spatial frequencies are processed more rapidly and accurately in the RVF than in the LVF (Christman, 1987; Hardyck, 1983; Jonsson \& Hellige, 1986; Michimata \& Hellige, 1987; Nevskaya \& Leushina, 1987; Sergent, 1982a, 1982b, 1982c, 1983a, 1983b, 1983c, 1985, 1987; Sergent \& Hellige, 1986).

Sergent (1982a, 1982b, 1982c, 1983a, 1983b, 1983c, $1985,1987)$ proposed that some tasks require low-spatialfrequency information for their successful completion. These tasks are performed better in the LVF if the stimuli contain the required low-frequency information because of the right cerebral hemisphere's superior ability at processing low spatial frequencies. Tasks that require high-spatial-frequency information are better performed when stimuli containing this necessary information are presented in the RVF. Sergent (1985), for instance, presented familiar faces at 100 -msec exposure durations in the RVF and LVF, and had subjects perform three tasks: (1) name the person as quickly as possible, (2) classify the person as professor or nonprofessor, and (3) classify the person as male or female. The subjects gave faster correct reaction times to faces presented in the RVF than to faces pres\&nted in the LVF on the tasks that required identification of the person ( 1 and 2 ). Lowpass filtering the faces to remove high spatial frequency produced an increase in reaction time, but more importantly, reaction time was faster in the LVF for all three tasks.

When only low spatial frequencies were available in the stimulus, it was necessary to perform the tasks on the basis of low-spatial-frequency information. The removal of high spatial frequencies from the stimuli had a large effect on the first and second tasks, because subjects were forced to perform the tasks on the basis of the low spatial frequencies in stimuli, but had little effect on the third task, because it was carried out on the basis of low spatial frequencies even when stimuli were unfiltered. These findings support the hypothesis that right-hemispheric superiority is found on tasks in which low spatial frequencies are both available in the stimulus and necessary for successful completion of the experimental task.

Although Sergent (1985) established that the results of visual-field experiments are related to the level of stimulus analysis necessary (task factors) and the type of information available in the internal representations of stimuli, it is not clear that spatial-frequency information itself is responsible for the pattern of results. Although low-pass filtering and the manipulation of luminance, retinal eccentricity, blur, and exposure duration prevent high spatial frequencies from being available in the internal representation of a stimulus, there is also less overall information available. The pattern of visual-field asymmetries described in this section could reflect the right hemisphere's relative resistance to information degradation (Michimata \& Hellige, 1987).

Having subjects perform different tasks is not the only way to access different cognitive operations. A visual stimulus undergoes transformations into different forms of representation. A sensory representation is based on the proximal stimulus, and the appropriate dimensions of this representation are expressed in degrees of visual angle or cycles per degree of spatial frequency. Our perceptual experience is based more on the properties of the object itself (the distal stimulus), and an accurate representation of the distal stimulus would have dimensions in feet, meters, or cycles per object. We infer, therefore, that tasks more dependent on the distal properties (cycles per object) operate on a higher stimulus representation than tasks dependent on the proximal properties (cycles per degree). This distinction between distal and proximal measures is illustrated in Figure 4, using letters that have each been digitally filtered to contain a 1 -octave range of spatial frequencies. If Figure 4 is viewed at a fixed distance, the filtered letters in each column contain the same center spatial frequency in cycles per degree, despite the fact that the letters are of three different sizes. Stimuli along each negative diagonal contain the same center spatial frequency in cycles per object although they differ in center cycles per degree. The number of cycles per object, unlike the number of cycles per degree, is invariant as a function of viewing distance.

Norman and Ehrlich (1987a) asked subjects to either detect or identify the orientation of horizontal and vertical sinusoidal gratings viewed from different distances. Reaction times to perform the detection task were determined by the proximal spatial frequency of the grating (cycles per degree). Reaction times to identify low spatial frequencies, however, were determined by the distal spatial-frequency content, and performance for higher spatial frequencies was determined by the proximal spatialfrequency content in cycles per degree.

Similar results were found with complex targets (Norman \& Ehrlich, 1987b). Subjects were shown pictures of tanks that had been digitally band-pass filtered to contain 2-octave ranges of spatial frequency, and were asked to identify each tank. Both reaction time and probability of correct response were determined by distal spatial frequency at relatively low center spatial frequencies, but this relationship broke down at higher frequencies.

\section{Method}

Subjects. Twenty-five University of Colorado undergraduates served as observers to fulfill a course requirement. All were righthanded, with normal or corrected-to-normal vision. 


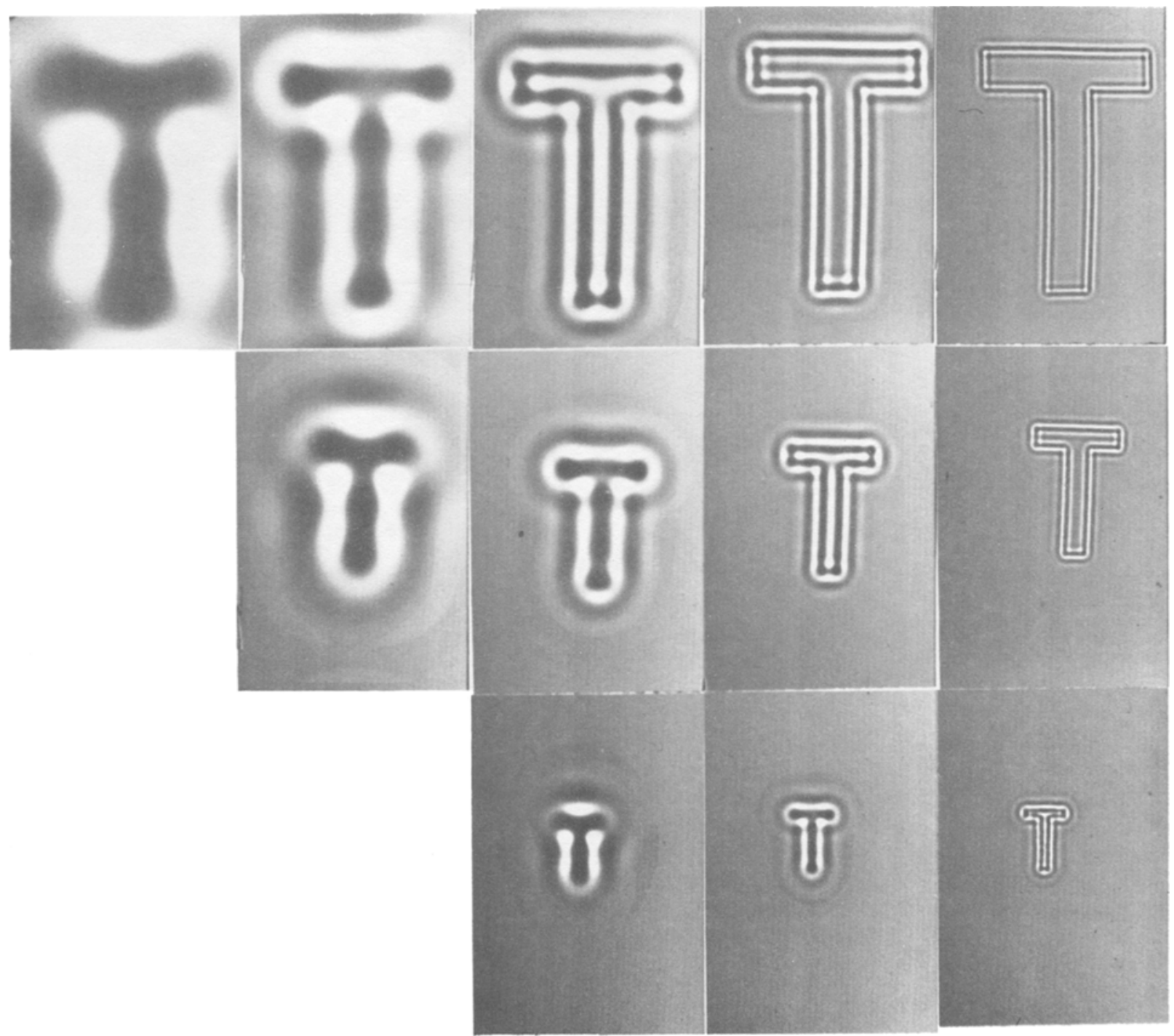

Figure 4. Band-pass filtered images of the letter $T$, equated for contrast. Small, medium, and large letters (bottom to top) subtended heights of $0.5^{\circ}, 1.0^{\circ}$, and $2.0^{\circ}$, and were filtered with 1-octave Gaussian ring filters with center spatial frequencies of $1,2,4,8$, and 16 cpd (left to right). In each vertical column, the stimuli have the same proximal spatial frequency; along a diagonal, the stimuli have the same distal spatial frequency.

Stimuli. The stimuli were based on the black, uppercase Letraset Futura Medium letters L, H, T, and F mounted on a white background, the same letters used by Sergent (1982b). There were 108 stimuli in all: 12 unfiltered letters (4 letters $\times 3$ sizes), 48 letters that had been Gaussian band-pass filtered to contain 1-octave ranges of spatial frequency but were unmatched for contrast, and 48 filtered letters which, additionally, were matched for equal peak-to-peak contrast of 0.30 . The actual stimuli were $35-\mathrm{mm}$ slides, with the innermost edge of each letter positioned in its slide mount so that it was $1.5^{\circ}$ to the left or right of a central fixation point (resulting in $2 \times 108$ slides). The filtered stimuli, matched in contrast, derived from the letter $T$ are shown in Figure 4.

The 48 filtered stimuli were produced by the following filtering operations. The three letter sizes were chosen so that the heights subtended in the actual experiment were $0.5^{\circ}, 1.0^{\circ}$, and $2.0^{\circ}$. Five filters, with proximal center spatial frequencies of 1.0, 2.0, 4.0, 8.0 , and 16.0 cycles per degree, were used. The $2.0^{\circ}$ letters were filtered with all five filters; the $1.0^{\circ}$ letters were filtered with the four highest filters; the $0.5^{\circ}$ letters were filtered with the three highest filters. These 12 filters were applied to each of the four letters. The appendix provides further details about the filtering operations.

Apparatus. Two channels of a three-channel projection tachistoscope were used to project the LVF and RVF stimuli. The third channel projected a $0.05^{\circ}$ fixation dot. The projectors were Kodak RA-960 random-access slide projectors fitted with Uniblitz shutters. The beams of the projectors were combined by means of halfsilvered mirrors and were directed to an opal glass screen mounted in the wall of the projector room. Presentation of stimuli and timing of the shutters was controlled by a BASIC program running on a Southwest Technical Products S/09 microcomputer system.

Procedure. The subject sat in a room adjacent to the projectors and viewed the opal glass screen binocularly at a distance of $122 \mathrm{~cm}$, with his/her head positioned by a foreheadrest. The fixation spot 
was always present except during stimulus presentation. The subject fixated the spot and then initiated a trial by pressing a button. Following a 500-msec delay, a stimulus was presented in the LVF or RVF for a duration of $150 \mathrm{msec}$. The letters $L$ and $H$ were defined as target letters, and $\mathrm{T}$ and $\mathrm{F}$ were nontarget letters. The subject pressed an appropriate response button with the right hand to indicate whether the stimulus was a target letter or a nontarget letter. The subjects were instructed to respond as quickly and as accurately as possible. Responses were collected by the computer and stored for later analysis.

The three stimulus sizes were presented in separate blocks of trials, with a different random block order for each subject. Before each block, the subjects had a practice session in which each stimulus appeared once in each visual field. The order of stimulus presentation was random, and each stimulus appeared 10 times within each block. Feedback was not provided during the trials.

\section{Results and Discussion}

Two measures of target classification were derived from our data: accuracy and reaction time. Accuracy measures based on percentages (e.g., hit rate and percent correct) are known to be biased by decision factors that are independent of perceptual sensitivity (Swets, 1986a, 1986b). The hit rates and false-alarm rates for target detection were therefore transformed into a detection accuracy measure $\left(d^{\prime}\right)$ and a decision bias measure (likelihood ratio, $\beta$ ) using the signal detection model based on Gaussian probability distributions with equal variance:

$$
\begin{aligned}
d^{\prime} & =z_{\mathrm{HR}}-z_{\mathrm{FAR}} \\
x_{\mathrm{c}} & =-z_{\mathrm{FAR}} \\
\beta & =f\left(x_{\mathrm{c}}\right)_{s n} / f\left(x_{\mathrm{c}}\right)_{n}
\end{aligned}
$$

where $z_{\mathrm{HR}}$ and $z_{\mathrm{FAR}}$ are the $z$-score transform of the hitrate and false-alarm-rate probabilities, $x_{c}$ is the critical value of the decision variable, and $f\left(x_{c}\right)_{s n}$ and $f\left(x_{c}\right)_{n}$ are the Gaussian probability densities at $x_{c}$ for the target distribution and the nontarget distribution, respectively (Green \& Swets, 1974). A $d^{\prime}$ of 0.0 indicates no targetdetection accuracy; a $\beta$ of 1.0 indicates an unbiased decision criterion. Reaction time was represented by the mean reaction time of correct responses (hits and correct rejections). The statistical analyses of $\beta$ were actually performed on the natural logarithm of $\beta$, since the distribution of $\beta$ is extremely skewed, ranging from zero to plus infinity, with a mode of 1.0 for an unbiased observer.

Unfiltered letters. The unfiltered letters were included to provide a baseline against which subjects' performance with the filtered stimuli could be compared. These data formed a $2 \times 3 \times 25$ within-subjects design ( 2 visual fields, 3 sizes, 25 subjects) embedded within the larger data set. The mean reaction time, accuracy, and decision criteria for classification of the three sizes of unfiltered letters are presented in Figure 5 for the LVF and RVF. Separate analyses of variance were computed for each of the three dependent variables, and the probability of making a Type I error was set at .01. Visual field of presentation had no significant effect on reaction time, accuracy,
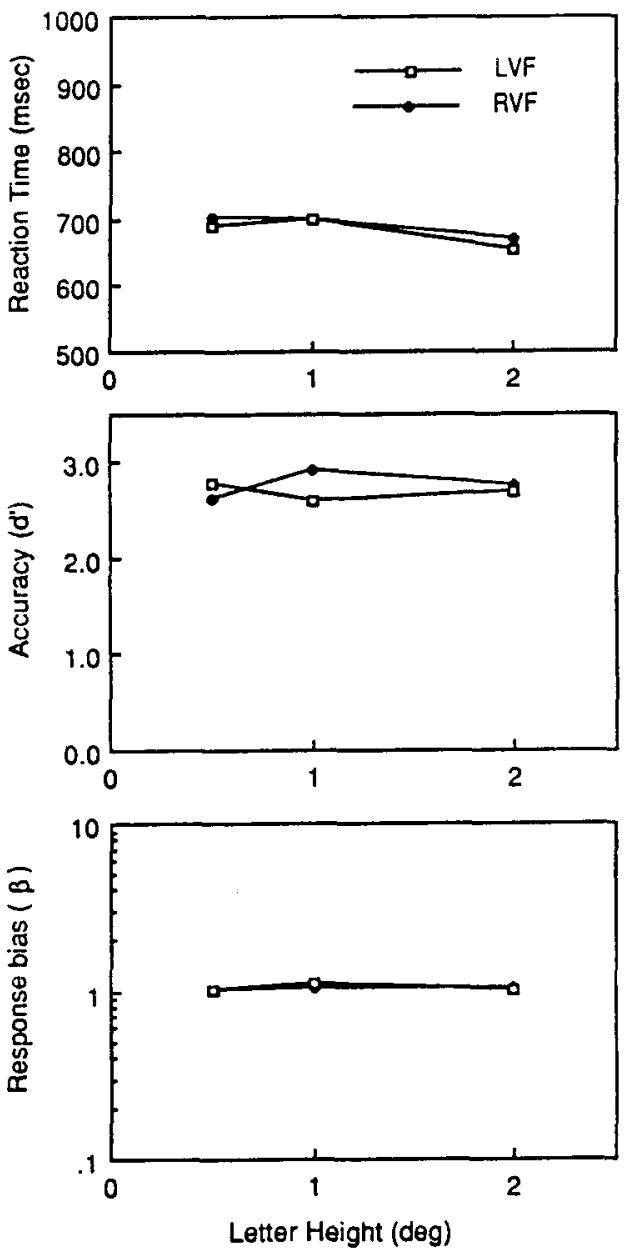

Figure 5. Performance on the target-classification task of Experiment 3 using unfiltered letters in the left (LVF) and right (RVF) visual fields as a function of letter height. Each point is the mean of 25 subjects. (Top) Mean reaction times for hits and correct rejections (msec). (Middle) Target-classification accuracy ( $\left.d^{\prime}\right)$. (Bottom) Likelihood ratio decision criterion $(\beta)$.

and decision criterion. Letter size likewise had no significant effect, and none of the interactions were significant.

Filtered letters. The effects of spatial-frequency filter, letter contrast, and letter size are presented in Figure 6 for mean correct reaction time, in Figure 7 for classification accuracy, and in Figure 8 for decision criterion. Since all five spatial-frequency filters were not combined with all three letter sizes, the data formed an incomplete factorial design. We were most interested to learn if different spatial-frequency bands contained in the visual stimuli affected performance differently when presented in the LVF or RVF. Such effects are represented by the filter frequency $\times$ visual field interaction in an analysis of variance. To test the effect of the experimental variables with an analysis of variance, only the stimuli produced by the 

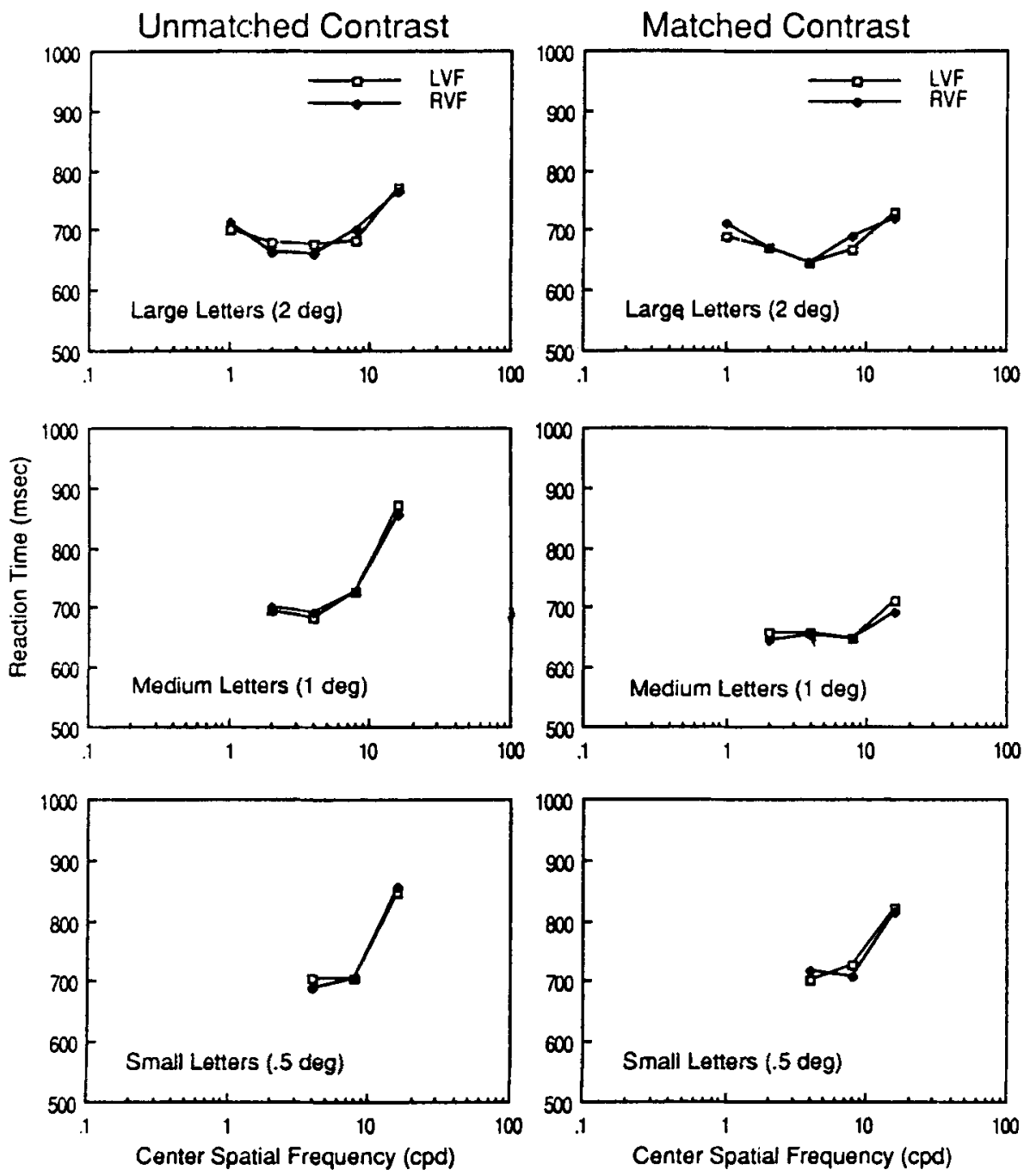

Figure 6. Mean reaction times for hits and correct rejections (msec) on the target-classification task of Experiment 3 using filtered letters unmatched in contrast (left column) and matched in contrast (right column) in the left (LVF) and right (RVF) visual fields as a function of filter center spatial frequency. Each point is the mean of 25 subjects. (Top) Letters $2.0^{\circ}$ high. (Middle) Letters $1.0^{\circ}$ high. (Bottom) Letters $0.5^{\circ}$ high.

three highest frequency filters were included as a main factor. This analysis was a $2 \times 2 \times 3 \times 3 \times 25$ withinsubjects design (visual field $\times$ contrast $\times$ filter frequency $X$ letter size $X$ subjects). As before, the probability of making a Type I error was set at .01.

Neither reaction time nor detection accuracy were significantly affected by visual field of presentation, nor were there any significant visual field $x$ filter spatial frequency interactions. Filter spatial frequency did significantly affect both reaction time $[F(2,48)=35.8]$ and accuracy $[F(2,48)=256.7]$, as might be concluded from the curvilinear functions shown in Figures 6 and 7. Equating the stimuli for contrast also improved reaction time $[F(1,24)$ $=32.6]$ and accuracy. $[F(1,24)=454.3]$. As can be seen in Figures 6 and 7, the improvement in performance brought about by equating the contrast of the stimuli occurred mostly at the highest spatial frequency, and as a result the interaction between contrast and filter frequency was significant for reaction time $[F(2,48)=13.62]$ and accuracy $[F(2,48)=149.6]$. Increasing letter size slightly, but significantly, increased accuracy $[F(2,48)=12.4]$ but did not influence reaction time.

The statistical analyses confirmed what can be seen in Figure 8: Subjects held a significantly higher decision criterion when they performed the target-classification task on stimuli presented in the RVF than when they did so on stimuli presented in the $\operatorname{LVF}[F(1,24)=15.0]$. None of the interactions of visual field with the other variables was significant. As filter frequency increased, decision criterion decreased significantly $[F(2,48)=7.97]$, although the effect depended on the letter size $[F(4,96)=$ $6.95]$ and stimulus contrast $[F(4,96)=5.52]$.

These findings mean that even though detection accuracy was equal in the two visual fields, subjects had 

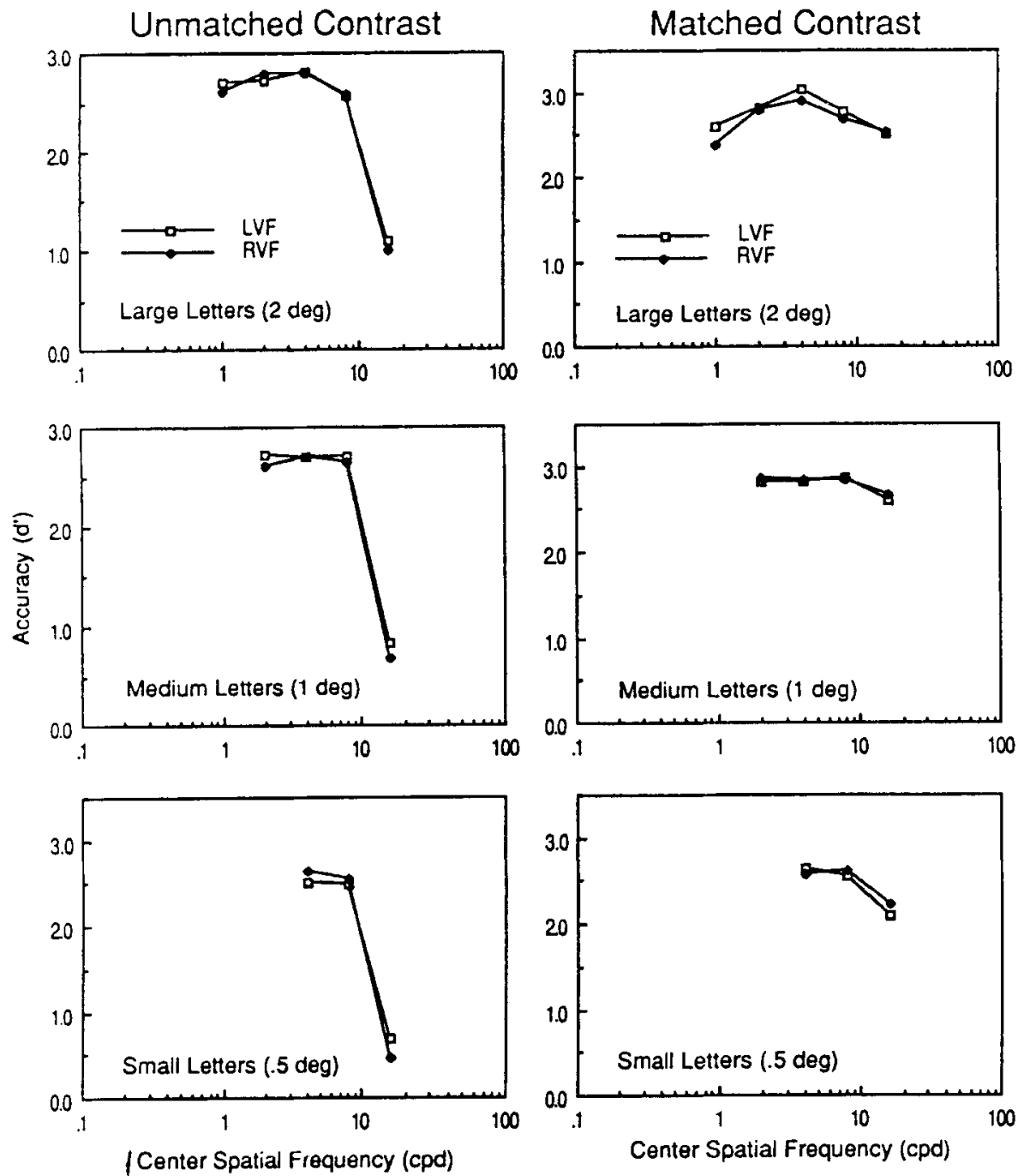

Figure 7. Accuracy $\left(d^{\prime}\right)$ on the target-classification task of Experiment 3 using filtered letters unmatched in contrast (left column) and matched in contrast (right column) in the left (LVF) and right (RVF) visual fields as a function of filter center spatial frequency. Each point is the mean of 25 subjects. (Top) Letters $2.0^{\circ}$ high. (Middle) Letters $1.0^{\circ}$ high. (Bottom) Letters $0.5^{\circ}$ high.

higher hit rates for stimuli in the LVF. This LVF superiority was entirely due to the different decision criteria used in the two visual fields. These effects are illustrated in Figure 9 , in which mean $\beta$, detection accuracy, and target hit rate are plotted as a function of filter frequency for the LVF and RVF and averaged across all other conditions.

Mean reaction time and detection accuracy for the three letter sizes, averaged across visual field and contrast, are plotted in Figure 10 as a function of proximal spatial frequency (cycles per degree) and distal spatial frequency (cycles per object). From inspection of Figure 10, we conclude that it is the proximal spatial-frequency content of the stimuli that influenced reaction time and detection accuracy, since the data from the three letter sizes are coincident when frequency is plotted in cycles per degree.
The hypothesis that hemispheric asymmetries are dependent on the spatial-frequency content of stimuli is not supported by our results, because no visual field effects, other than response criterion, and no visual-field $\times$ spatial frequency interactions were found. We find that the hemispheres are differentially affected by information degradation, independent of spatial frequency, and that observers use different response criteria when processing stimuli in the LVF and RVF. Although response criterion is uninfluenced by visual field of presentation when stimuli are unfiltered, observers hold a higher decision criterion for stimuli presented in the RVF than they do for stimuli presented in the LVF when stimulus information content is reduced by spatial frequency filtering. The higher decision criterion means that more information or more certainty is required with presentation of degraded 

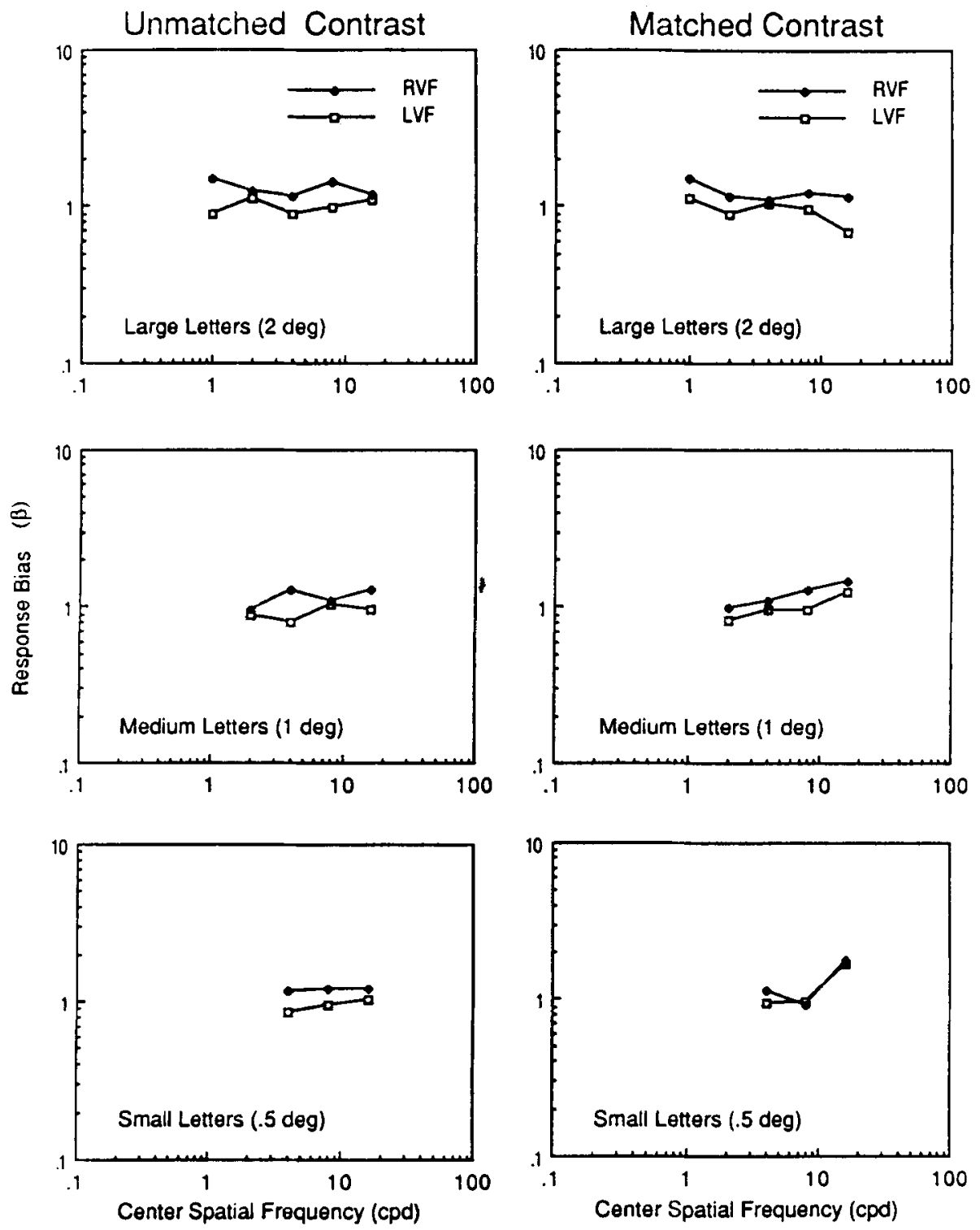

Figure 8. Likelihood ratio decision criterion $(\beta)$ on the target-classification task of Experiment 3 using filtered letters unmatched in contrast (left column) and matched in contrast (right column) in the left (LVF) and right (RVF) visual fields as a function of filter center spatial frequency. Each point is the mean of 25 subjects. (Top) Letters $2.0^{\circ}$ high. (Middle) Letters $1.0^{\circ}$ high. (Bottom) Letters $0.5^{\circ}$ high.

stimuli in the LVF in order to make the decision that the target letters are present.

This experiment differs from most studies of hemispheric performance in that bias-free detection accuracy $\left(d^{\prime}\right)$ and response criterion $(\beta)$ were computed from the data. Our finding, illustrated in Figure 9, that observers performing tasks with filtered stimuli presented in the LVF and RVF are using different decision criteria, suggests that it is important to use bias-free measures of performance. Had hit rate been used as an index of accuracy, we would have concluded that the LVF was superior to the RVF for the detection of filtered letters, a conclusion unwarranted in the context of signal detection theory.

Because the RVF was biased, relative to the left, to respond that the stimulus presented was not a target, we could label it "conservative" and the other "liberal." Whether the differences in hemispheric response bias we report here represent (a) a true conservative versus liberal bias or (b) a less generalizable bias to respond " $T$ " or " $F$ " versus " $L$ " or " $H$ " is an unanswered empirical question that needs to be addressed by future research. In this light, it is interesting to note that the finding that the LVF consistently has a lower decision criterion than 

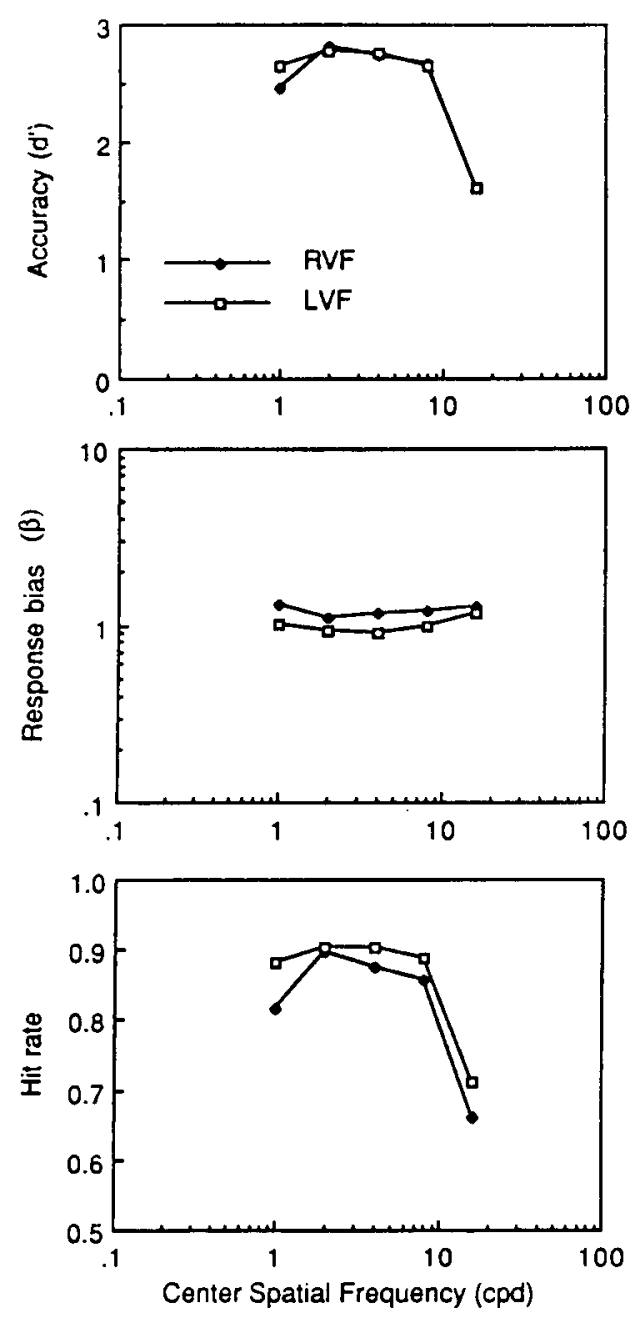

Figure 9. Performance on the target-classification task of Experiment 3 using filtered letters in the left (LVF) and right (RVF) visual fields as a function of filter spatial frequency. Each point is the mean of 25 subjects, two contrasts, and three stimulus sizes. (Top) Targetclassification accuracy $\left(d^{\prime}\right)$. (Middle) Likelihood ratio decision criterion $(\beta)$. (Bottom) Hit rate for classification of target letters.

the RVF agrees with that of Chiarello, Senehi, and Soulier (1986) for a lexical decision task. Their subjects were biased to give a "word" response to stimuli in the RVF and biased to give a "nonword" response to stimuli in the LVF. But, the key point is that we have determined that our results are due to a response bias. It would be incorrect to conclude that one hemisphere is more competent than the other at completing the task.

One possible objection to our conclusions, which was raised by one of the reviewers of this paper, is that the asymmetries we found in Experiment 3 are sensory and not cognitive in nature; there are differences in the spatialfrequency contents of letters that cause the sensory signals of the target and nontarget letters to differ. Although it is almost certain that there are differences in spatial- frequency contents of target and nontarget letters, this has nothing to do with whether our results are attributable to sensory or cognitive processes. The fact that the obtained visual-field asymmetries are attributable to response bias means that our results must not be sensory in nature; response bias is, by definition and according to signal detection theory, a decision process that acts upon, and is independent of, a sensory signal (Green \& Swets, 1974). Additionally, the fact that the cognitive process of classification must occur prior to a response makes our results represent, to some extent, cognitive operations rather than simple sensory detection.

It might be argued that the failure to find any visual field $x$ spatial frequency interaction may be due to the choice of task or stimulus parameters. It has been suggested, as noted in the introduction to this experiment, that a number of stimulus and task parameters interact with spatial frequency to determine the magnitude and direction of cerebral laterality effects. Consequently, it could be argued, following Sergent (1983a), that hemispheric differences for spatial frequency processing do exist, but show up only when task performance is resource-limited (Norman \& Bobrow, 1975), since hemispheric asymmetries often occur only when tasks are resource-limited (see Friedman \& Polson, 1981, for a review). Because most subjects in the present experiment found the task easy, it may be that they had cognitive loads insufficient to produce a visual field $\times$ spatial frequency interaction. It could also be argued that the use of a relatively long stimulus duration of $150 \mathrm{msec}$ and a suprathreshold contrast of .3 somehow counteracted the influence of spatial frequency filtering.

One might correctly suggest that in order to fully address such criticisms, we would need to perform a more comprehensive study in which several different tasks are performed on the same set of stimuli, as in the study by Sergent (1985) that was described in the introduction to Experiment 3. A variety of input factors would have to be manipulated, as well. These input factors would have to include variables such as retinal eccentricity, stimulus contrast, luminance, size, exposure duration, contrast, and filter bandwidth, in addition to mean spatial frequency. If spatial frequency is, in fact, one of the critical variables that visual-field asymmetries depend upon, then performing this more comprehensive assessment of task by input factors should yield results that would demonstrate the significant relationship between spatial frequency and visual field.

In answer to these possible objections and suggestions, we note that we have found exactly what previous researchers have found. When stimuli are degraded, either by being blurred, by reducing their exposure durations or contrasts, and so forth, or in the case of Experiment 3, by being digitally filtered, then detection hit rate becomes lower in the RVF than in the LVF. As we noted earlier, this general finding is the primary source of evidence cited in favor of Sergent's hypothesis regarding spatial frequen- 

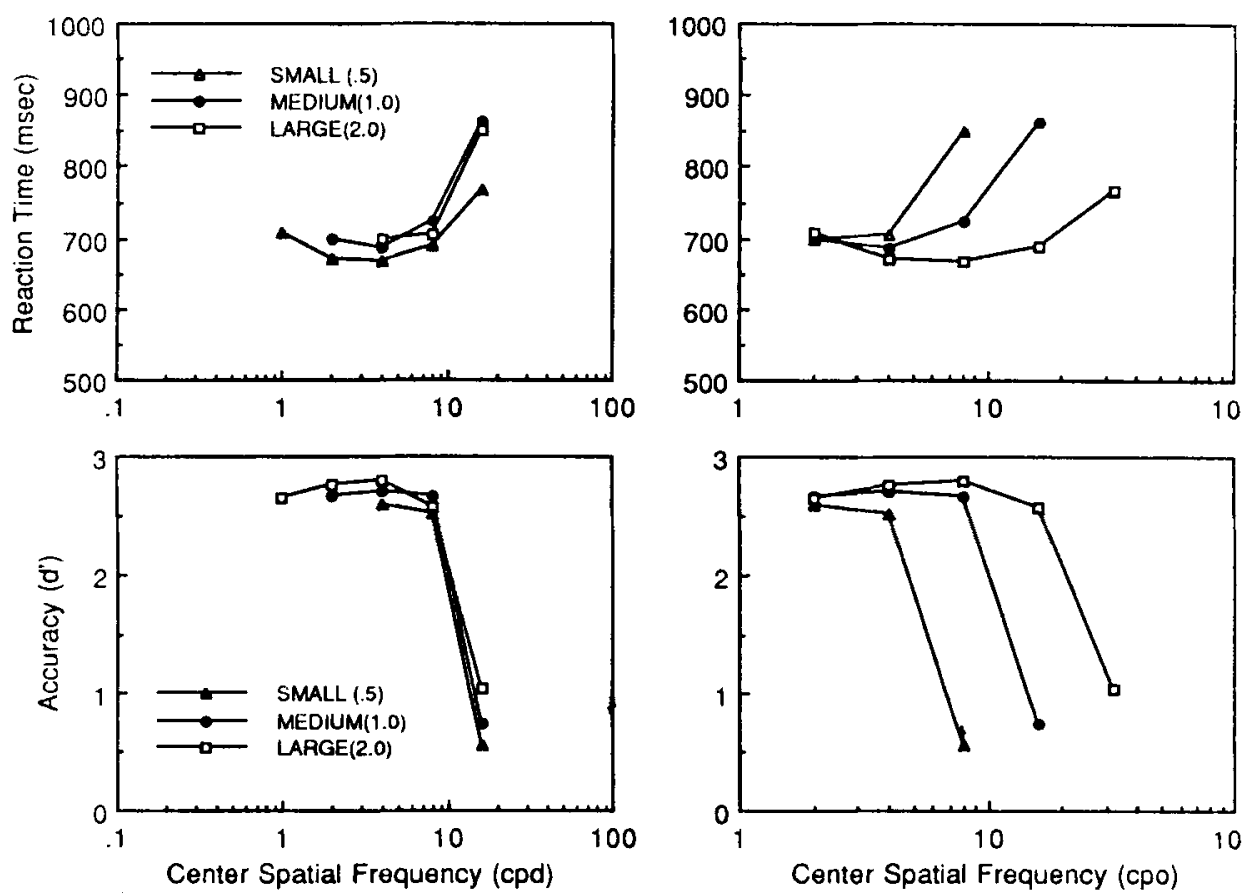

Figure 10. Mean correct reaction time (top) and mean classification accuracy (bottom) as a function of proximal spatial frequency in cycles per degree (left column) and of distal spatial frequency in cycles per object (right column) for letters $0.5^{\circ}, 1.0^{\circ}$, and $2.0^{\circ}$ in height.

cies and the hemispheres, and we have replicated it, as shown in Figure 9. We have replicated it using tasks that are very similar to those used previously (Jonsson \& Hellige, 1986; Sergent, 1982b), so it is unclear to us why the tasks in these previous experiments may have been more resource-limited than the task in the present experiment.

In all the previous reports, however, the effects of spatial frequency were confounded with the degree of stimulus degradation, and the effects of response bias were not examined. In this, the only study in which spatial frequency is not confounded with these other variables, these other variables can account for the visual-field asymmetries. So, the suggestion that we have somehow counteracted the effects of spatial frequency seems unlikely to us. A more plausible hypothesis is that a relation between spatial frequency and hemispheric asymmetry does not exist, and that the results from previous published reports, like the results from Experiment 3, can be explained in terms of hemispheric response bias and general level of stimulus degradation. Given the fact that this plausible alternative hypothesis exists and can explain previous findings as well as our own, we believe that there is currently no solid empirical support for the hypothesis regarding spatial frequencies and the hemispheres, and also believe that a more comprehensive examination of task and input factors would not support this particular hypothesis.

This is not to suggest that a more comprehensive analysis of the type advocated by Sergent and Hellige (1986; Hellige \& Sergent, 1986) would not be quite valuable. Although our results do not point to a relationship between hemispheric asymmetry and spatial frequency, they do not contradict the earlier findings that input and task factors are, in fact, related to hemispheric asymmetry. The question of which input and task factors are most strongly related to the direction and magnitude of observed asymmetries remains of considerable interest.

\section{GENERAL DISCUSSION}

Several researchers have suggested that visual-field asymmetries are influenced by the spatial-frequency content of stimuli (Christman, 1987; Hardyck, 1983; Hellige et al., 1984; Jonsson \& Hellige, 1986; Nevskaya \& Leushina, 1987; Rao, 1979; Rao et al., 1981; Sergent, 1982a, 1982b, 1982c, 1983a, 1983b, 1983c, 1985, 1987; Sergent \& Hellige, 1986). In the present study, and contrary to this suggestion, we find bilateral symmetry for spatial frequency processing for contrast sensitivity (Experiment 1), visible persistence (Experiment 2), and speed and accuracy of letter classification (Experiment 3). In Experiment 3, asymmetries are observed in the decision criteria $(\beta)$ used in judging filtered stimuli. Observers hold a higher criterion for stimuli presented in the RVF.

The present data are in agreement with the widely held hypothesis that the hemispheres are functionally symmetrical at the earliest (sensory) stages of information processing and that hemispheric asymmetries emerge at later (cognitive) stages (Moscovitch, 1979; Sergent, 1983a, 1983c). Visual-field asymmetries are found in Experiment 3, in which a letter classification task was performed, but not in Experiments 1 and 2, in which sen- 
sory detection alone was required to complete the task. Findings of hemispheric symmetry for spatial frequency processing do not necessarily preclude other sensory-level explanations of hemispheric asymmetry. Recent work indicates a LVF advantage for phase processing and/or the processing of local image components (Fiorentini \& Berardi, 1984; Rentschler, Christen, Christen, \& Landis, 1986).

The results of the third experiment are of special interest because they are inconsistent with the widely cited hypothesis that hemispheric asymmetries are dependent upon spatial-frequency content for certain cognitive operations, including letter classification (Chiarello, Senehi, \& Soulier, 1986; Christman, 1987; Hardyck, 1983; Hellige et al., 1984; Jonsson \& Hellige, 1986; Nevskaya \& Leushina, 1987; Sergent, 1982a, 1982b, 1982c, 1983a, 1983b, 1983c, 1985, 1987; Sergent \& Hellige, 1986). Our results lead us to reject this hypothesis.

Results from Experiment 3 are consistent with those of previous studies. As noted above, a considerable amount of research indicates that various manipulations that reduce the perceptual quality of visual stimuli impair performance more when the stimuli are presented in the RVF than when they are presented in the LVF. We find no hemispheric asymmetries in performance when unfiltered stimuli are presented, but when the perceptual quality of the stimuli is reduced by band-pass filtering, detection hit rate is lower in the RVF than in the LVF, as shown in Figure 9. In this sense, we have obtained the same shift in performance that others have reported. Our results, however, are not explainable in terms of spatial frequency or hemispheric specialization, but rather in terms of information degradation and hemispheric response bias.

In Experiment 3, we have demonstrated that performance asymmetries are due to response bias, and we rule out hemispheric differences in the processing of spatial frequencies as an explanation of the results because we manipulated spatial-frequency content directly. We conclude that our results, and possibly the results of others, are not attributable to differences in hemispheric specialization for the processing of different spatial frequencies but, rather, to differences in hemispheric response bias. The response biases of the left and right hemispheres are dependent upon the level of information degradation.

\section{REFERENCES}

BADCOCK, D., \& LoVEgrove, W. (1981). The effects of contrast, stimulus duration, and spatial frequency on visible persistence in normal and specifically disabled readers. Journal of Experimental Psychology: Human Perception \& Performance, 7, 495-505.

BeAton, A., BLAKEMORE, C. (1981). Orientation selectivity of the human visual system as a function of retinal eccentricity and visual hemifield. Perception, 10, 273-282.

BlaKe, R., \& Mills, J. (1979). Pattern and flicker detection examined in terms of the nasal-temporal division of the retina. Perception, 8 , 549-555.

Bowling, A., \& Lovegrove, W. (1980). The effect of stimulus dura- tion on the persistence of gratings. Perception \& Psychophysics, 27, 574-578.

Bowling, A., Lovegrove, W., \& Mapperson, B. (1979). The effects of spatial frequency and contrast on visual persistence. Perception, 8, 529-539.

Brigham, E. O. (1974). The fast Fourier transform. Englewood Cliffs, NJ: Prentice-Hall.

BRYDEN, M. P. (1982). Laterality: Functional asymmetry in the intact brain. New York: Academic Press.

Chiarello, C., Senehi, J., Soulier, M. (1986). Viewing conditions and hemisphere asymmetry for the lexical decision. Neuropsychologia, 24, 521-529.

Christman, S. D. (1987). Effects of perceptual quality on hemispheric asymmetries in visible persistence. Perception \& Psychophysics, 41, 367-374.

Delis, D. C., Robertson, L. C., Efron, R. (1986). Hemispheric specialization of memory for visual hierarchical stimuli. Neuropsychologia, 24, 205-214.

DI LoLLO, V. (1981). Hemispheric symmetry in duration of visible persistence. Perception \& Psychophysics, 29, 21-25.

Di LoLLo, V. (1983). On laterality of visual aftereffects: A rejoinder. Perception \& Psychophysics, 33, 599-603.

FIELD, D. J. (1987). Relations between the statistics of natural images and the response properties of cortical cells. Journal of the Optical Society of America A, 4, 2379-2394.

FIORENTINI, A., \& BERARDI, N. (1984). Right-hemisphere superiority in the discrimination of spatial phase. Perception, 13, 695-708.

Friedman, A., \& Polson, M. C. (1981). Hemispheres as independent resource systems: Limited-capacity processing and cerebral specialization. Journal of Experimental Psychology: Human Perception \& Performance, 7, 1031-1058.

GREEN, D. M., \& SweTs, J. A. (1974). Signal detection theory and psychophysics. New York: Krieger.

HARDYCK, C. D. (1983). Seeing each other's point of view: Visual perceptual lateralization. In J. B. Hellige (Ed.), Cerebral hemisphere asymmetry: Method, theory, and application (pp. 219-254). New York: Praeger.

HARVEY, L. O., JR. (1986). Visual memory: What is remembered? In F. Klix \& H. Hagendorf (Eds.), Human memory and cognitive capabilities (pp. 173-187). New York: North-Holland/Elsevier.

Heluge, J. B. (1983). Hemisphere $x$ task interaction and the study of laterality. In J. B. Hellige (Ed.), Cerebral hemisphere asymmetry: Method, theory, and application (pp. 411-443). New York: Praeger.

Hellige, J. B., Corwin, W. H., \& Jonsson, J. E. (1984). Effects of perceptual quality on the processing of human faces presented to the left and right cerebral hemispheres. Journal of Experimental Psychology: Human Perception \& Performance, 10, 90-107.

Hellige, J. B., \& Sergent, J. (1986). Role of task factors in visual field asymmetries. Brain \& Cognition, 5, 200-222.

Jonsson, J. E., HeLlige, J. B. (1986). Lateralized effects of blurring: A test of the visual spatial frequency model of cerebral asymmetry. Neuropsychologia, 24, 351-362.

KitTerle, F. L. (1986). Psychophysics of lateral tachistoscopic presentation. Brain \& Cognition, 5, 131-162.

KITTERLE, F. L., KAYE, R. S. (1985). Hemispheric symmetry in contrast and orientation sensitivity. Perception \& Psychophysics, 37 391-396.

Meyer, G. E., \& Maguire, W. M. (1977). Spatial frequency and the mediation of short term visual storage. Science, 198, 524-525.

Michimata, C., \& Hellige, J. B. (1987). Effects of blurring and stimulus size on the lateralized processing of nonverbal stimuli. Neuropsychologia, 25, 397-407.

Moscovitch, M. (1979). Information processing in the cerebral hemispheres. In M. S. Gazzaniga (Ed.), Handbook of behavioral neurobiology: Vol. 2, Neuropsychology (pp. 379-446). New York: Plenum.

Nevskaya, A. A., \& Leushina, L. I. (1987). Hemispheric differences in the perception of spatial-frequency-filtered objects. Perception, 14, 256 (A31) 
Norman, D. A., \& Bobrow, D. G. (1975). On data-limited and resource-limited processes. Cognitive Psychology, 7, 44-64.

Norman, J., \& EHRLich, S. (1987a). Sensory and higher-level processes in the detection and identification of sine-wave gratings (IPDM Report No. 46). Haifa, Israel: University of Haifa, Institute of Information Processing and Decision Making.

Norman, J., \& Ehrlich, S. (1987b). Spatial frequency filtering and target identification. Vision Research, 27, 87-96.

RAo, S. M. (1979). Cerebral laterality: Spatio-temporal frequency discrimination in the right and left visual fields. Unpublished doctoral dissertation, Wayne State University.

Rao, S. M., Rourke, D., \& Whitman, R. D. (1981). Spatio-temporal discrimination of frequency in the right and left visual fields: A preliminary report. Perceptual \& Motor Skills, 53, 311-316.

Rentschler, I., Christen, L., Christen, S., \& Landis, T. (1986). Features versus spatial phase in a tachistoscopic laterality experiment. Perception \& Psychophysics, 39, 205-209.

Rusdijk, J. P., Kroon, J. N., \& Van der Wildt, G. J. (1980). Contrast sensitivity as a function of position on the retina. Vision Research, 20, 235-241.

ROSE, D. (1983). An investigation into hemisphere differences in adaptation to contrast. Perception \& Psychophysics, 34, 89-95.

SERGENT, J. (1982a). Basic determinants in visual-field effects with special reference to the Hannay et al. (1981) study. Brain \& Language, 16, 158-164.

SERGENT, J. (1982b). The cerebral balance of power: Confrontation or cooperation? Joumal of Experimental Psychology: Human Perception \& Performance, 8, 253-272.

SERGENT, J. (1982c). Methodological and theoretical consequences of variations in exposure duration in visual laterality studies. Perception \& Psychophysics, 31, 451-461.

Sergent, J. (1983a). The effects of sensory limitations on hemispheric processing. Canadian Journal of Psychology, 37, 345-366.

SERGENT, J. (1983b). Hemispheric competence and perceptual confusability. Quarterly Journal of Experimental Psychology, 35A, 589-596.

SERGENT, J. (1983c). Influence of task and input factors on hemispheric involvement in face processing. Psychological Bulletin, 93, 481-512.

SERGENT, J. (1985). Influence of task and input factors on hemispheric involvement in face processing. Journal of Experimental Psychology: Human Perception \& Performance, 11, 846-861.

SERGENT, J. (1987). Failures to confirm the spatial-frequency hypothesis: Fatal blow or healthy complication? Canadian Journal of Psychology, 41, 412-428.

SERGENT, J., \& Hellige, J. B. (1986). Role of input factors in visualfield asymmetries. Brain \& Cognition, 5, 174-199.

SPRINGER, S. P., \& DEUTSCH, G. (1985). Left brain, right brain. New York: Freeman.

SwETS, J. A. (1986a). Form of empirical ROCs in discrimination and diagnostic tasks: Implications for theory and measurement of performance. Psychological Bulletin, 99, 181-198.

SwETs, J. A. (1986b). Indices of discrimination or diagnostic accuracy: Their ROCs and implied models. Psychological Bulletin, 99, 100-117.

Szelag, E., Budohoska, W., \& KoltusKa, B. (1987). Hemispheric differences in the perception of gratings. Bulletin of the Psychonomic Society, 25, 95-98.

Taylor, M. M., \& Creelman, C. D. (1967). PEST: Efficient esti- mates on probability functions. Journal of the Acoustical Society of America, 74, 782-787.

Vassilev, A., VersKaya, A. A., Manahilov, V., Mitov, D., \& Leushina, L. I. (1985). Spatial vision in the left and right visual fields. Perception, 14, A38.

\section{APPENDIX}

\section{Digital Image Processing}

Each of the 12 Letraset letters ( 4 letters $\times 3$ sizes) was digitized into a $256 \times 256$ image with 256 gray levels, using a Colorado Video Model 274c frame store controlled by a Southwest Technical Products S/09 microcomputer. The gray levels were adjusted so that the minimum pixel level was 95 and the maximum level was 160 . The digitized images were transferred to the VAX-11/780 computer in the Department of Psychology's Computer Laboratory for Instruction in Psychological Research (CLIPR). The two-dimensional fast Fourier transform (Brigham, 1974) of each stimulus was then computed. The resulting frequency spectra were amplitude filtered with annular band-pass filters (isotropic in orientation) with a 1-octave Gaussian bandpass frequency profile (symmetrical in log spatial frequency). These types of filters were used in a previous experiment and were chosen because they were related to the known properties of the human visual system (Harvey, 1986).

Five filters with center spatial frequencies of $2.78,5.57,11.1$, 22.3 , and 44.6 cycles per image were used. Since a $256 \times 256$ image subtended $2.78^{\circ}$ of visual angle in height, these filters had the following proximal center frequencies: 1.0, 2.0, 4.0, 8.0, and 16.0 cycles per degree. Band-pass filtering at high spatial frequencies reduces the contrast in the filtered image because the spatial-frequency amplitude of most natural images is inversely proportional to frequency (Field, 1987). The second set of filtered images (contrast-matched) was created by adjusting the gray scale so that they all had a peak-to-peak contrast of 0.30 .

Each stimulus was displayed as a negative image surrounded by a uniform gray field equal to the mean luminance of the images on an Electrohome V10-430 monochrome video monitor. The letter was then shifted left or right on the screen so that when positioned in the film frame and viewed by the subject in the experiment, its innermost edge was positioned $1.5^{\circ}$ to the left or right of a central fixation point. The screen was then photographed using Kodak Panatomic X fine-grain black-andwhite $35-\mathrm{mm}$ film. The resulting positives were then mounted as $35-\mathrm{mm}$ slides.

(Manuscript received July 27, 1988; revision accepted for publication April 24, 1989.) 